This item was submitted to Loughborough's Research Repository by the author.

Items in Figshare are protected by copyright, with all rights reserved, unless otherwise indicated.

\title{
Customisable 3D printed microfluidics for integrated analysis and optimisation
}

PLEASE CITE THE PUBLISHED VERSION

http://dx.doi.org/10.1039/C6LC00562D

PUBLISHER

(C) Royal Society of Chemistry

VERSION

AM (Accepted Manuscript)

\section{PUBLISHER STATEMENT}

This work is made available according to the conditions of the Creative Commons Attribution-NonCommercialNoDerivatives 4.0 International (CC BY-NC-ND 4.0) licence. Full details of this licence are available at: https://creativecommons.org/licenses/by-nc-nd/4.0/

\section{LICENCE}

CC BY-NC-ND 4.0

\section{REPOSITORY RECORD}

Monaghan, Tom, Matthew Harding, Russell A. Harris, Ross J. Friel, and Steven Christie. 2016. "Customisable 3D Printed Microfluidics for Integrated Analysis and Optimisation". Loughborough University. https://hdl.handle.net/2134/22219. 


\title{
Lab-on-a-Chip
}

\section{ARTICLE}

\section{Customisable 3D Printed Microfluidics for Integrated Analysis and Optimisation}

Received 00th January 20xx Accepted 00th January 2 DOI: $10.1039 / \times 0 \times x 00000 \times$

www.rsc.org/

\author{
T. Monaghan ${ }^{\text {a }}$, M. J. Harding ${ }^{\text {b }}$, R. A. Harris ${ }^{c}$ and R. J. Friel ${ }^{a}$, S. D. R. Christie ${ }^{b^{*}}$
}

The formation of smart Lab-on-a-Chip (LOC) devices featuring integrated sensing optics is currently hindered by convoluted and expensive manufacturing procedures. In this work, a series of 3D-Printed LOC devices were designed and manufactured via stereolithography (SL) in a matter of hours. The spectroscopic performance of a variety of optical fibre combinations were tested, and the optimum path length for performing Ultraviolet-Visible (UV-Vis) spectroscopy determined. The information gained in these trials was then used in a reaction optimisation for the formation of carvone semicarbazone. The production of high resolution surface channels $(100-500 \mu \mathrm{m})$ means that these devices were capable of handling a wide range of concentrations $(9 \mu \mathrm{M}-38 \mathrm{mM})$, and are ideally suited to both analyte detection and process optimisation. This ability to tailor the chip design and its integrated features as a direct result of the reaction being assessed, at such a low time and cost penalty greatly increases the user's ability to optimise both their device and reaction. As a result of the information gained in this investigation, we are able to report the first instance of a 3D-Printed LOC device with fully integrated, in-line monitoring capabilities via the use of embedded optical fibres capable of performing UV-Vis spectroscopy directly inside micro channels.

\section{Introduction}

During the past decade, Lab-on-a-Chip (LOC) technology has seen revived research interest from the chemical and process industry as an efficient tool for chemical synthesis. ${ }^{1-4}$ Whilst the merits of microfluidic devices based on their advantages have been well documented, there is a continuous desire for the integration of analytical tools within these systems for online process monitoring. 5 , The ability to perform in-line reaction monitoring makes LOC technology a powerful laboratory tool for reaction and kinetic studies.

Monolithic integration of analytical sensing devices such as optical fibres directly into the channels of LOC devices has the potential to provide true online analysis of the reaction mixture. Aside from fast data acquisition, online reaction monitoring has the potential to make complex analysis simpler to perform and permits faster and more reliable process optimization. A large proportion of the work published regarding the integration of spectroscopic equipment within LOC devices has centred on absorbance measurements via Ultraviolet-Visible (UV-Vis) spectroscopy in order to identify

${ }^{a}$ Wolfson School of Mechanical and Manufacturing Engineering, Loughborough University, Ashby Road, Loughborough, LE11 3TU, United Kingdom. E-mail: t.monaghan@lboro.ac.uk

${ }^{b}$ Department of Chemistry, Loughborough University, Epinal Way, Loughborough, LE11 3TU, United Kingdom. E-mail: s.d.christie@lboro.ac.uk

c. School of Mechanical Engineering, University of Leeds, Leeds, LS2 9JT

† Electronic Supplementary Information (ESI) available: Details regarding Design of Experiments, materials testing and further device characterisation are included in the supplementary information. See DOI: $10.1039 / \times 0 \times x 00000 \times$ the composition and concentration of the sample analyte. ${ }^{7,8}$ Under favourable optical configurations, UV-Vis absorption spectroscopy has been shown capable of achieving detection limits in the $\mu$-molar range. ${ }^{9}$ These techniques are often noninvasive and provide high degrees of both temporal and spatial resolution. ${ }^{10,11}$

Whilst numerous groups have reported the use of UV-Vis for product quantification during an organic reaction and several others for process monitoring (e.g. residence time, sample distribution and mixing) via external flow-through cells, this set-up is still not as ideal as in-situ integration where spectroscopic measurements are focused directly inside the microchannel. ${ }^{12-14}$ Furthermore, only a single instance of embedded optical fibres being used for direct in-line reaction monitoring and optimisation via UV-Vis spectroscopy has been reported. ${ }^{15}$ This lack of further publishing likely arises as a result of the requirement to integrate UV transparent waveguides into the channels of the device. ${ }^{16}$ This requirement not only significantly increases the complexity and cost of the LOC devices when produced using traditional manufacturing methods, but also leads to large path lengths and therefore limits the working concentration range which can be used in these devices.

In situations where rapid concept-to-chip time is a priority, or multiple design iterations of a similar device are required traditional multistage manufacturing methods such as photolithography become less ideal. ${ }^{17}$ These classical microfabrication techniques have been shown to support wide range of LOC configurations and functions ${ }^{18-20}$, however they also require specialised resources and technical training 
beyond most end users of the LOC devices. This not only limits the end user to potentially less than optimal designs, it also makes the production of multiple design iterations prohibitively expensive and time consuming for most research situations.

In contrast to traditional manufacturing techniques, Additive Manufacturing (AM), or 3D-Printing (3DP) has demonstrated the ability to allow users the opportunity to produce bespoke micro- and milli-fluidic reactors, sometimes with multiple design iterations, and allow the user total control over the design of the fluidic network in $3 \mathrm{D} \cdot{ }^{18,21-24} 3 \mathrm{DP}$, is a layer by layer manufacturing approach in which $3 \mathrm{D}$ objects can be formed from an initial computer-aided design (CAD) model in a matter of minutes or hours. Compared to the lithographic techniques often employed in microfluidic device manufacture, 3DP offers a much simpler fabrication process by foregoing the need to use a master for replica moulding. ${ }^{17,25}$ Whilst many of the polymer based materials used in 3DP are not ideal for high temperature, high pressure synthetic procedures as a result of their mechanical properties, they are able to tolerate a range of organic solvents at moderate temperatures for extended periods. ${ }^{26,27}$

In this work, we have created high resolution surface channels via Stereolithography (SL), to produce the first example of a 3D printed LOC device featuring directly embedded optical fibres. Through this approach, multiple design iterations were produced in a single run of the Stereolithography Apparatus (SLA), allowing for a thorough assessment of the chips optimal design. As a result of the information gained in this investigation, we are able to report the first instance of a stereolithography produced LOC device with capable of performing UV-Vis spectroscopy directly inside the micro channels, in real time for use in both in-line monitoring and detection.

\section{Methodology}

\section{Equipment}

All samples produced in this work were manufactured using a 3D Systems Viper Si2 Stereolithography system (3D Systems Inc., South Carolina, USA). This machine features an integrated digital signal processor (DSP) controlled high speed scanning system with a single, solid-state UV laser capable of delivering $100 \mathrm{~mW}$ in power. All parts were manufactured using the machines pre-set High Resolution (HR) mode which employs a beam diameter of $75 \mu \mathrm{m}$ and a step height (layer thickness) of $50 \mu \mathrm{m}$.

\section{Materials}

The optically clear Accura ${ }^{\circledR} 60$ resin (3D Systems Inc South Carolina, USA) was employed in this work. This resin contained a cycloaliphatic diepoxide, polymerised by a photo-cationic initiator, and an aliphatic tetra-acrylate, polymerised by a photo-radical initiator. ${ }^{28}$ It has also previously been demonstrated to be able to cope with a range of organic solvents by Capel et al. $^{26}$
Table 1. Information regarding optical fibres used in embedding trails

\begin{tabular}{|l|cccc|}
\hline & $\begin{array}{c}\text { Core } \\
\text { Diameter, } \\
\mu \mathrm{m}\end{array}$ & $\begin{array}{c}\text { Cladding } \\
\text { Diameter, } \\
\mu \mathrm{m}\end{array}$ & $\begin{array}{c}\text { Coating } \\
\text { Diameter, } \mu \mathrm{m}\end{array}$ & $\begin{array}{c}\text { Wavelength, } \\
\mathrm{nm}\end{array}$ \\
\hline Fibre A & $50 \pm 2 \%$ & $125 \pm 1 \mu \mathrm{m}$ & $250 \mu \mathrm{m} \pm 5 \%$ & $250-1200 \mathrm{~nm}$ \\
Fibre B & $105 \pm 2 \%$ & $125 \pm 1 \mu \mathrm{m}$ & $250 \mu \mathrm{m} \pm 5 \%$ & $250-1200 \mathrm{~nm}$ \\
Fibre C & $50 \pm 2 \%$ & $125 \pm 1 \mu \mathrm{m}$ & $250 \mu \mathrm{m} \pm 5 \%$ & $180-1200 \mathrm{~nm}$ \\
Fibre D & $100 \pm 2 \%$ & $125 \pm 1 \mu \mathrm{m}$ & $250 \mu \mathrm{m} \pm 5 \%$ & $180-1200 \mathrm{~nm}$ \\
\hline
\end{tabular}

The optical fibres chosen for embedding in this work were various specifications of multimode optical fibre (MMOF) Table 1 . The reason to include multimode optical fibres as opposed to single mode optical fibres (SMOF) was a direct result of their respective core sizes. The larger cores sizes associated with MMOF's result not only in increased sensitivities, but also relaxed alignment tolerances which results in easier manufacturability/fibre alignment.

It is important to note that a large variety of optical fibres with various core sizes and wavelengths are available with the same total diameter as the ones used in this work. As a result they could be directly transposed for the fibres implemented here, should the proceeding reaction require a different spectra range. All of the fibres used in this work are commonly used and commercially available with diameters typical of most multimode optical fibres. This freedom is a significant advantage for those looking to tailor their device to a specific reaction/wavelength and is currently not possible using current traditional methods whereby the incorporated fibres are aligned using expensive optical alignment equipment and then permanently fixed.

All sealing of microfluidic devices was achieved using Tesa 4965 pressure sensitive adhesive (PSA) film (Tesa UK Ltd., Milton Keynes, UK) and backed with a sheet of Lexan 8010 Polycarbonate Film (Cadillac Plastics LTD, Swindon, UK). This choice was based upon data gathered in prior systematic studies (ESI).

\section{Device manufacture and characterisation}

Each SL - Resin combination has the potential to produce different characteristics in regards to the resolution of the parts it produces. This is a result of the various additives contained within different resins e.g. monomers, initiators and inhibitors, and how these interact with the laser system used to cure them. Therefore in order to assess the potential size of features produce via the particular SL - Resin combination and the geometric constraints of these features, a resolution test device containing an array of various sized features was produced; recessed L-shaped square micro-channels (25-300 $\mu \mathrm{m})$, recessed square profiles $(25-500 \mu \mathrm{m})$, recessed square trenches separated by varying distances $(62.5-1000 \mu \mathrm{m})$ and a series of raised square features $(50-500 \mu \mathrm{m})$ to establish the normalised axial resolution limits. All features were built to a depth of height of $500 \mu \mathrm{m}$. It was hoped that the production of these small features would prove useful when designing structures such as small dimension channels, multiple z-plane fluidic channels and chaotic mixers. It was of particular interest
Comment [SS1]: Reviewer 2: comment 2

Comment [SS2]: Reviewer 1 commen on being the first to publish this type of work

Comment [SS3]: Reviewer 1: address their comment regarding the shape of the channels not being described till later on

Comment [SS4]: Reviewer 3 commen 2 on axial feature resolution

Comment [SS5]: Reviewer 1 commen 6 regarding the depth of through holes 
to determine the minimum channel size it was possible to produce and if/how far the produced part deviated from the initial CAD model. This was achieved through a combination of Scanning Electron Microscopy (SEM) and the open source, Java-based image-processing program ImageJ 1.x, developed at the National Institutes of Health (Maryland, USA).

\section{Optical Fibre Integration}

A large proportion of prior works published regarding the integration of spectroscopic equipment within LOC devices has centred on absorbance measurements via UV-Vis spectroscopy in order to identify the composition and concentration of the sample analyte. ${ }^{7,8,15}$ A common approach used in the microfluidics industry in order to achieve these measurements is based upon the housing of two optical fibres in a perpendicular configuration to the plane of the fluidic channel, which is transparent to either UV or Vis or both, in order to guide the incident light through the sample. This transmitted light is then collected by the opposing waveguide in order to yield absorbance information. ${ }^{15,29,30}$ This method requires precise optical alignment which must be achieved via tailormade micro-channels to house these fibres. Using traditional manufacturing methods, there is a requirement for UV-Vis transparent windows to be incorporated into the device as most materials used for the construction of LOC devices inhibit wavelengths below $\sim 300-350 \mathrm{~nm}$ (e.g., silicon, glass). This acts to further confound and complicate the manufacturing process, leading to increased device cost as well as increasing path length and system light losses. It was hoped that through direct fibre incorporation via custom manufactured alignment guides, direct reaction monitoring would be possible within the AM devices without the requirement to include UV-Vis transparent waveguides.

\section{Fibre Alignment Guide Optimisation}

The geometry and size of the fibre alignment guides was considered to be a vital design consideration for the incorporation of optical fibres within the high resolution SL microfluidic device. In order to ensure that the device was capable of dealing with the pressures required for flow chemistry, the fibre-channel combination should match closely in geometry. This ensures the fibre is tightly sealed within the channel and therefore reduces any leaking hazards. The relationship between the shape and size of predefined fibre alignment guides on the ability to accommodate optical fibres under an applied adhesive film was therefore systematically investigated.

A CAD model was constructed featuring several U-shaped channel geometries at three different sets of dimensions Figure 1. The size of these different grooves were chosen based on the size of the fibres to be embedded, and varied as it was not known if there would be any contraction of the part during curing, and to also account for in-tolerance variations of the diameter of the fibre - Table $1 . \mathrm{SL}$ was then implemented in order to construct the samples containing the channel

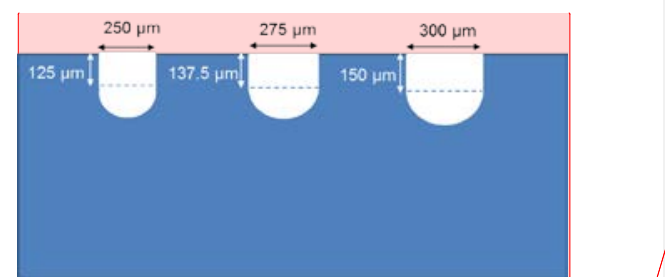

Figure 1. U-shaped fibre channels at the various selected dimensions

variations, prior to equipping them with the commercially available optical fibres.

Once the fibres were placed into the different grooves, the channels were sealed using the adhesive tape. The SL components were then polished to a $0.05 \mu \mathrm{m}$ finish and imaged using a Leica DM6000 optical microscope setup. Optical microscopy measurements were used as a means of selecting the most suitable fibre guide geometry. This was established by determining which channel geometry produced the closest fit to the profile of the fibre and which fibre combination recorded the high number of spectral counts. Additional testing to establish if any leaking was apparent at high flow rates was performed by infusing a dye solution into the chosen fibre guide geometry. This was performed using an aqueous solution of methylene blue $(0.25 \mathrm{M})$ and a flow rate of $1 \mathrm{~mL} / \mathrm{min}$.

By removing the requirement for these UV transparent waveguides between the light source and the channel, there is potential to reduce the path length between the fibres thereby reducing light losses in the system and hence improve the sensitivity of the device to both upper and lower ends of the concentration range. There is also a significant reduction in cost associated with this change in manufacturing procedure. This work acted to highlight the additional functionality that can be imparted as a result of the design freedom, iterative design and low cost associated with SL. ${ }^{31}$

\section{UV-Vis Cell Manufacture and Optimisation}

\section{Optical Fibre Combination Testing}

Due to the advantages afforded by AM in the development of LOC devices, a simple test device was designed and quickly manufactured, directly from the CAD data, in order to establish which combination of optical fibre core sizes (Fibres $A$ and $B$, Table 1) produced the highest degree of light transmission across rectangular microchannel with a fixed distance $(500 \mu \mathrm{m})$. The manufactured cell featured three prealigned groves within which these different combinations of fibres could be aligned and placed - Figure 2 (a). As a result of the rapid and low cost nature of these devices, these combinations could be tested in series, on the same device platform, and without the need for costly or time consuming modelling software or the excessive costs associated with iterative design using traditional manufacturing methods.
Comment [SS6]: Image enlarged for clarity

Comment [SS7]: Reviewer 1's third comment on testing cells with a dye to check for any sign of leaking

Comment [SS8]: Reviewer 1's comment regarding the shape of the channel not being mentioned early on 

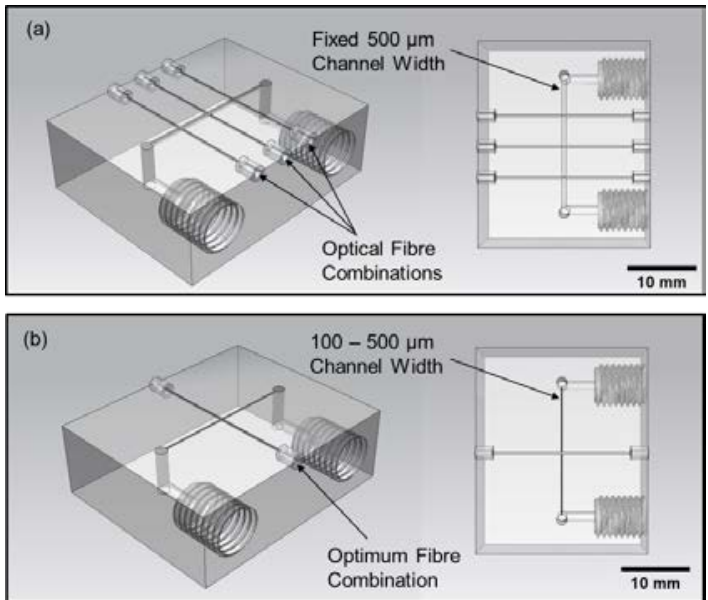

Figure 2. CAD models of (a) optical fibre combination test device and (b) path length test device

The fibres were placed into the groves with the aid of an optical microscope system (Leica MZ125) ensuring the tips of the fibres were positioned in line with the wall of the channel which ran perpendicular to the fibres axial direction. These channels were then sealed using the selected PSA laminate.

Once manufactured, the transmission performance of the optical combinations within the device was established. In order to achieve this, the embedded optical fibres were connected to a deuterium-halogen light source (DH-2000, Ocean Optics, Oxford, UK) and an S2000 UV-Vis spectrometer with a USB1000-ADC analogue to digital converter (Ocean Optics) via the use of bare fibre adapters (multi-mode SMA connector, Newport Spectra-Physics Ltd, Oxford, UK). A measure of the light transmitted across the channel was then recorded at $265 \mathrm{~nm}$ for 10 and 100 ms intervals for each fibre combination to establish any potential differences in light transmission of the various combinations as a result of a change in core diameter. This wavelength was chosen specifically to relate to later work in in-line reaction monitoring. This time period ensured that a high enough number of counts were recorded by the detector to illuminate any potential difference between combinations, but not so high as to saturate the detector.

\section{Optimal Path Length for Spectroscopic Measurements}

Through the manufacture of a device featuring significantly different fluidic channels widths and therefore path lengths from the transmitting optics to the collecting optics, it is possible to determine at which point this absorption flattening effect occurred and thereby establish a working concentration range for these devices. In light of this a device featuring a single, pre-aligned central groove for housing the chosen optical fibre combination was fabricated and equipped with the most successful of the fibre combinations determined previously. The ends of these fibres were then intersected by a central fluidic channel of various diameters - Figure 2 (b). The width of this channel was varied from $100-500 \mu \mathrm{m}$ in $100 \mu \mathrm{m}$ increments.

The spectroscopic performance of the cells was then tested by analysing aqueous nicotinamide solutions (Acros, 99\% purity), a UV-active water soluble B-vitamin. Using a value of $2650 \mathrm{~L} \mathrm{~mol}^{-1} \mathrm{~cm}^{-1}$ for the molar absorptivity, $\varepsilon$, [NIST Standard Reference Database, No. 69] the appropriate concentration range to test the cells was determined by the Beer-Lambert law - Equation $1 .^{32}$ This compound was chosen as it has a relatively low value of molar absorptivity and is therefore this technology will likely be applicable to a wide range of organic compounds.

Equation 1

$$
A=\log \frac{I_{o}}{I}=\varepsilon c L
$$

\section{A = Absorbance (A.U.) \\ $\mathrm{I}_{\mathrm{o}}=$ Incident Light Intensity \\ I = Transmitted Light Intensity \\ $\varepsilon=$ Molar Absorptivity $\left(\mathrm{L} \mathrm{mol}^{-1} \mathrm{~cm}^{-1}\right)$ \\ $\mathrm{c}=$ Sample Concentration $\left(\mathrm{mol} \mathrm{L}^{-1}\right)$ \\ $\mathrm{L}=$ Path Length $(\mathrm{cm})$}

Solutions were therefore prepared from approximately 0.5 to $25 \mathrm{mM}$ to provide a thorough working range. The uppe detection limit of the cell was of equal importance to the lower detection limit for flow chemistry where maxima throughput is achieved by using as high a working concentration as possible with the constraint of minimising solvent consumption. The higher concentration nicotinamide solutions should therefore identify the linear dynamic range for each path length and determine which of them was most practically useful in reaction monitoring and optimisation.

The prepared solutions were pumped into the device, starting with the lowest concentration, and rinsed with water between each solution to ensure minimal carry over. This process was repeated for each of the path lengths produced and the optimal path length determined through an assessment of the available working range. The acquisition times were varied so that approximately 3000 counts were recorded at $260 \mathrm{~nm}$ with water in the cell. This number of counts again ensured that a sufficient intensity of light was recorded by the detector without any saturation occurring. 10 averaged spectra were used for each measurement and all spectra were recorded with 3 point boxcar averaging.

In order to estimate the lowest concentrations which could be analysed using this cell, a compound with a high molar attenuation coefficient was utilised to determine the cells limit of detection (LOD). The compound chosen for these measurements was fluorescein, a synthetic water soluble organic compound with a molar attenuation coefficient of $76900 \mathrm{~L} \mathrm{~mol}^{-1} \mathrm{~cm}^{-1}$. A dilution series was then formed from 0.01 to $1.78 \mathrm{mM}$ and the LOD for each cell then determined using Equation $2 .^{33}$
Comment [SC9]: Selected what? Comment [SS10]: New image to better show the individual test pieces 
Equation 2. $\quad$ LOD $=$ regression intercept $+3 s_{B}$

$$
\text { Where; } \quad s_{B}=\sqrt{\sum_{i} \frac{\left(y_{i}-\hat{y}_{i}\right)^{2}}{n-2}}
$$

$y_{i}=$ absorbance value (A.U.)

$\hat{y}_{i}=$ 'fitted' absorbance value (A.U.)

$\mathrm{n}=$ number of samples

\section{Use of UV-Vis Cell for Online Reaction Monitoring and Optimisation}

To test the suitability of the cells for online analysis of an organic reaction, the synthesis of carvone semicarbazone was performed in flow according to Figure 3 . This scheme consists of a condensation reaction between carvone and semi carbazide to form the carvone semicarbazone product. The product shows a slight shift in absorbance compared to the carvone starting materials of approximately $260 \mathrm{~nm}$ versus 245 $\mathrm{nm}$ respectively. Due to the small path lengths achievable with this manufacturing technique a full spectrum from 220-290 nm could be acquired, allowing the evolution of the carvone semicarbazone product to be monitored via UV-Vis spectroscopy due to a shift in the $\lambda_{\max }$ of the system as well as an increase in absorbance at $265 \mathrm{~nm}$.

An optimisation of conditions was performed through a response surface methodology (RSM), central composite faced (CCF) design of experiments (DOE) approach and continuous monitoring of the reaction UV spectrum via the integrated optical fibres. A total of two factors and three levels were employed to optimise the reaction conditions with respect to semicarbazone production. The relevant factors chosen for optimization of this reaction were the temperature of the reaction mixture and the residence time-Table 2 .

The reaction was performed using a Flowsyn flow chemistry platform (Uniqsis Ltd, Cambridge, UK). Separate reagent streams were mixed at a $T$ piece and a $5 \mathrm{~mL}$ stainless steel reaction coil was fitted immediately after. This system utilised the inbuilt chip heater platform as a means of reagent temperature control.

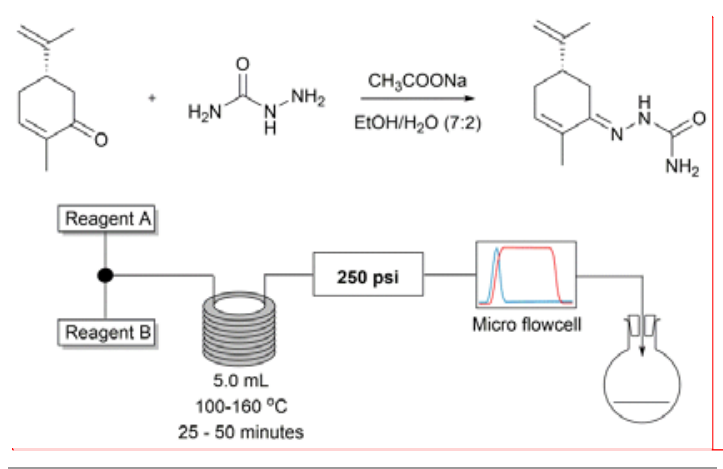

Figure 3. (above) reaction scheme for the formation of carvone semicarbazone (below) schematic for the flow setup
Table 2. DOE factors and levels used in the optimisation of carvone semicarbazone

\begin{tabular}{|c|ccc|}
\hline & \multicolumn{3}{|c|}{ Value } \\
\hline Temperature, ${ }^{\circ} \mathrm{C}$ & 120 & 140 & 160 \\
Residence Time, $\min$ & 25 & 33.3 & 50 \\
\hline
\end{tabular}

As it was intended to heat the proceeding reaction above the boiling point of the ethanol:water (7:2) solvent, a 200 PSI backpressure regulator (BPR) was installed after the stainless steel reactor to ensure that the reaction mixture remained liquid. In this instance, both starting materials were infused through two separate inlets (Inlets A and B). Reagent vial A was charged with carvone $(60 \mathrm{mM})$ whilst reagent vial $B$ was charged with semi carbazide ( $80 \mathrm{mM})$.

Spectra were collected when steady state conditions had been reached and all spectra were processed by min-max normalisation between 0 and 1 A.U. over the wavelength range 210 - $290 \mathrm{~nm}$. This normalisation offsets baseline disturbance that can arise as a result of the production of gas bubbles and background noise. The $265 \mathrm{~nm}$ absorbance value after normalisation was then taken as the response for the DOE.

A new 3DP flow cell featuring a $100 \mu \mathrm{m}$ spectroscopic path length was connected directly after the BPR via a $10 \mathrm{~cm}$ length

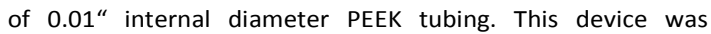
designed to fully demonstrate the $3 D$ capabilities of this technology and featured a complex split-and-recombine internal channel network. In order to monitor the reaction, the solution was then routed to the surface as was the case with previous flow cells. The cell was equipped with two solarisation resistant fibres (Fibre $C$ and D, Table 1 ) due to the need to operate at $<300 \mathrm{~nm}$ for long periods of time. The ability to change the fibres embedded as a result of the spectra range required indicates the level of potential for this technique. The acquisition time was $100 \mathrm{~ms}$ and 3 points were used for boxcar smoothing. In total 10 spectra were averaged for each acquisition.

\section{Results and Discussion}

\section{Device Manufacture and Characterisation}

The decision to pursue the manufacturing of microfluidic channels on the surface of SLA LOC devices was taken to avoid issues associated with the removal of uncured resin from fluidic channels whilst also removing unwanted channel distortion. In this vein, only square channels were investigated as they avoid the issues associated with 'stair stepping', a common feature in small AM structures which leads to rough surface textures on the unsupported walls. This effect is amplified when producing features at an angle to the scanning laser beam e.g. circular channels. This production of square channels is not only more practical based on the manufacturing processes; it also made alignment of the fibres with the walls of the channels substantially easier and may act to significantly reduce the degree of turbulence within the flow regime. Additional SEM images are included within the
Comment [SS11]: Reviewer 3: Comment 3 - Scheme resolution enhanced and schematic of flow setup included

Comment [SS12]: Reviewer 2 
ESI to highlight this stair stepping effect and its minimisation in square channels. These channel geometries have also been shown to be highly suitable for later UV-Vis monitoring applications. ${ }^{34}$ This effect leads to a rough surface texture on the unsupported walls of SL builds. In order to evaluate this, a number of basic positive and negative structures that may prove useful in LOC devices were manufactured on the surface of a SL wafer and analysed via SEM - Figure 4.

Resulting micrographs demonstrated that the 3DP SLA surface technique was able to produce channels much smaller than are achievable if the channels were formed internally (due to issues with clearing the uncured resin and channel quality). ${ }^{23,35}$ Observation of the channel cross-section demonstrated that no significant distortions or roughness was observed as is the case with most internal channel networks. Furthermore, there channels were capable of producing stable laminar flow regimes (See ESI). The features sizes achieved in this work mean that through the use of 3DP, and more specifically SLA, microfluidic devices with features sizes comparable to traditional manufacturing methods can now be produced. These channel sizes facilitate much more complex and functional parts due to the high degree of resolution, which are not possible using internal SLA channels. This will only serve to increase the complexity and potential for these

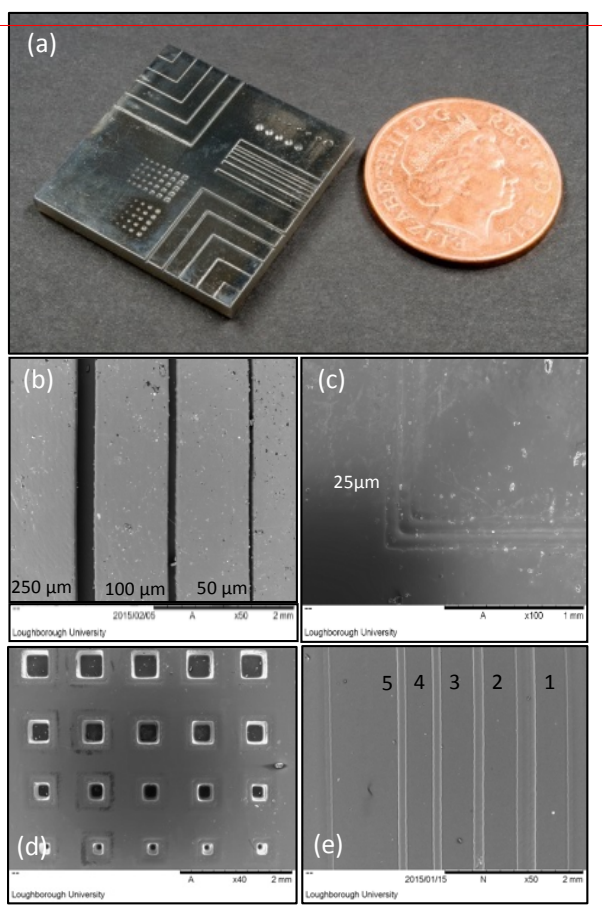

Figure 4. (a) Test piece produced in order to assess the resolution of 3DP-LOC devices and SEM images of (b) Successfully cleared channels from $50-250 \mu \mathrm{m}$ (c) unsuccessfully cleared $25 \mu \mathrm{m}$ channels (d) Successful manufacture of holes from 200-500 $\mu \mathrm{m}$ (e) SEM micrograph of channel seperation (raised channel wal sections appear lighter in colour) devices in future works such as fluid manipulation and biphasic flows. Images of a hydrodynamic focussing device have been included within the ESI for this work in order to highlight such possibilities. The minimum channel size successfully formed and cleared of resin was the $50 \mu \mathrm{m}$ channel - Figure 4 (b).

Below this $50 \mu \mathrm{m}$ threshold, the curing of resin material within the $25 \mu \mathrm{m}$ channels was visible in Figure $4-\mathrm{c}$ ). This was due to the lateral curing area being so large relative to the channel, that material within the channel itself was unintentionally cured. This occurs was as a direct result of the beam size used to the cure the resin and the photosensitivity of the polymer. The micrographs showed good channel quality with sharp features and no significant deviations along the channel path. As there was no covering layer/roof to these channels, the decision was made to produce all these test pieces with the channels on the top surface. The measured widths of all the channels produced using this method were within $10 \%$ of their modelled width, with some as low as $1 \%$ deviation in the case of the $1000 \mu \mathrm{m}$ channel. This quality and reproducibility is something that is not seen when forming internal channels via $\mathrm{SL}$.

A second important feature that was assessed was the minimum channel separation possible in these 3DP-LOC devices. The ability to densely pack fluidic channels into a device can be essential for providing sufficient residence times for reactants and lead to increasing production rates. To this end, a series of recessed $500 \mu \mathrm{m}$ channels were formed and separated by decreasing distances from 1000 to $25 \mu \mathrm{m}$. The details of these channels and the result of their testing are displayed in Table 3. Figure 4 (e) shows a labelled SEM micrograph of the channels formed.

As Table 3 indicates, the $62.5 \mu \mathrm{m}$ separation distances yielded a channel, however they were larger than the intended channel separation. Upon measurement of these features, the distances were noted as being $c a .100 \mu \mathrm{m}$ in both cases. This would indicate that the minimum channel separation is approximately $100 \mu \mathrm{m}$, which was a sufficient separation distance to allow for the dense packing of fluidic channel networks within a LOC device. This channel width minima was due to both the size of the laser beam 'spot' diameter used to cure the material $(75+15 \mu \mathrm{m})$ as well as the unintentional curing of additional material in the local area, due to the inherent photosensitivity of the polymer.

The final assessment made was centred on the ability to produce small features such as through holes for fluid transport in the $z$ axis and raised square features, which was seen as key in the fabrication of truly 3D LOC devices. In this sense, a device can be formed when long path lengths of internal features are formed, with the reactant mixture travelling to the surface, where the channel quality is significantly better, for measurement/analysis - Figure 4 (d). Through-holes were successfully manufactured in a range of sizes from 500-100 $\mu \mathrm{m}$ with no significant deviation in size and/or shape. Again however, when travelling below $100 \mu \mathrm{m}$ the shape of the hole became distorted, from a square to a spherical shape as well as the size, with holes typically
Comment [SS13]: Reviewer 2

Comment [SS17]: Reviewer 2

Comment [SS14]: Reviewer 2

Comment [SS15]: Images enlarged and combined for clarity, addressing reviewer

1 's comment regarding this figure
Comment [SS16]: Reviewers 1 comment 9 - he couldn't see which part of the image were channels 
Table 3. Channel dimensions and separation distances

\begin{tabular}{|c|ccc|}
\hline & $\begin{array}{c}\text { Channel Width, } \\
\mu \mathrm{m}\end{array}$ & $\begin{array}{c}\text { Distance to next } \\
\text { channel in CAD Model, } \\
\mu \mathrm{m}\end{array}$ & $\begin{array}{c}\text { Measured } \\
\text { Width on Test } \\
\text { Piece, } \mu \mathrm{m}\end{array}$ \\
\hline 1 & $500 \mu \mathrm{m}$ & $1000 \mu \mathrm{m}$ & $949 \pm 2.64$ \\
2 & $500 \mu \mathrm{m}$ & $500 \mu \mathrm{m}$ & $494 \pm 2.10$ \\
3 & $500 \mu \mathrm{m}$ & $250 \mu \mathrm{m}$ & $258 \pm 1.94$ \\
4 & $500 \mu \mathrm{m}$ & $125 \mu \mathrm{m}$ & $130 \pm 4.49$ \\
5 & $500 \mu \mathrm{m}$ & $62.5 \mu \mathrm{m}$ & $104 \pm 0.84$ \\
\hline
\end{tabular}

measuring around $100 \mu \mathrm{m}$. This is as a result of the spherical nature of the beam and also its size relative to the hole causing a rounding off of the features and over-curing of material in the local area due to the UV-effected zone produced by the laser. In regards to raised axial feature resolution, with the exception of the $50 \mu \mathrm{m}$ features, all structures matched with the initial CAD design. These smaller features however were measured at $c a .75-100 \mu \mathrm{m}$. This was attributed to the beam diameter and over-curing of resin local to the features.

The ability to form very intricate networks of fluidic channels onto the surface of relatively cheap devices, and furthermore, form these into 3D devices at minimal time or cost penalty is a significant advance in the production of LOC devices. This added design freedom can allow the user to create milli/micro feature size devices in a variety of complex designs depending on their needs. Additionally, the ability to fabricate multiple iterations of a similar design in order to optimise a reaction is something that usually cannot be investigated due to the relatively high cost of traditional LOC devices. In this situation a user could take information gained from an initial trial, and use that data to enhance and optimise both the design of their device and in turn, the reaction performed. It is these small feature sizes that will enable the ability to perform in-line, in-channel reaction monitoring which can be optimised through the use of multiple design iterations.

\section{Insertion of Optical Fibres into Predefined Channels}

The effect of both the size and geometry of pre-defined grooves on the quality of the seal produced when using PSA tapes to encapsulated optical fibres (Fibre B, Table 1) was investigated through microscopic analysis. The size of these different guides were chosen based on the size of the fibres to be embedded and to account for tolerances in the diameter of the fibre and the size of the channels in respect to the CAD design.

Optical microscopy performed on mounted cross-sections of the SL parts allowed for the best fibre-channel combination to be determined. This was defined as the combination which produced the most accurate alignment and tightest fibre/device fit. This was performed with not only visual inspection of the polished surface, but also through dye injection testing - Figure 5. Through this microscopic investigation it was determined that the most suitable geometry and size for the incorporation of optics within the SL part was that of a U-shaped channel with a width of $275 \mu \mathrm{m}$ and a $387.5 \mu \mathrm{m}$ depth - Figure 5 (a). This dark field image
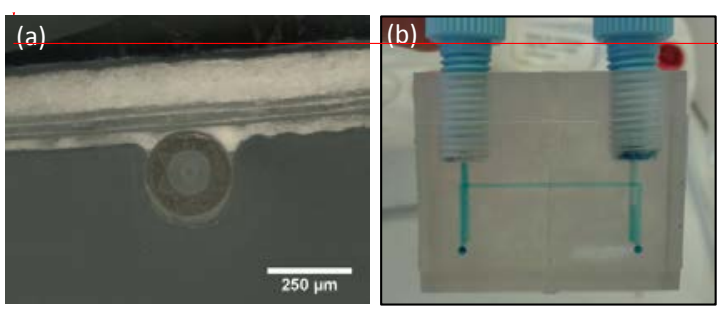

Figure 5. (a) $275 \mu \mathrm{m}$ u-shaped fibre guides and (b) dye testing of device fitted with fibre to ensure its robustness and ability to cope with high flow rates without leakage

highlights how empty space around the fibre appears to be filled in through the redistribution of adhesive from the overlying tape to produce a tight fit around the fibre, reducing any potential for leakage of aqueous media from the channels.

Any channels smaller than that shown in Figure 5 (a) were shown to produce a slight bulge of the fibre from the channel, whilst the larger channels resulted in a small degree of empty space around the fibre. Both of these situations can result in leaking of solvent from the channel and lead to penetrating underneath the cover layer, potentially leading to delamination of the covering layer. Additional micrographs of these fibre-guide combinations are located in the supplementary information for this work. Confirmation that the selected fibre guide geometry was suitable for later experiments was done through the injection of an aqueous dye solution. This testing indicated that no leakage from the channel into the intersection fibre guide had occurred - Figure 5 (b).

Through the manufacture of custom shape alignment guides, the system avoids the use of expensive optical alignment equipment, reducing the fabrication cost and complexity. Furthermore, due to the close match between the geometry of the fibre and the alignment groove, there is no requirement to integrate UV transparent waveguides into the device as is the case with previous works. ${ }^{16}$ This again reduces the cost and complexity and therefore increases the accessibility of this technology to research environments.

\section{Optical Fibre Combination Testing}

A test device was designed and manufactured in a matter of hours, and equipped with fibres of various core sizes and combinations. This device was then used to determine which combination of optical fibres produced the highest degree of light transmission (ESI). Figure 2 (a) details the key features of the flow cell whilst the details of the fibres are located in Table 1. Analysis of spectra produced using this device showed that fibre pairs with the same core diameters $(50-50 \mu \mathrm{m}$ and 105 $-105 \mu \mathrm{m}$ ) demonstrated lower transmission than pairs where mismatched core diameters were used (e.g. $50-105 \mu \mathrm{m}$ ). It was hypothesised that this was a result of the more efficient projection of light from a smaller fibre onto the face of the larger opposing optical fibre or conversely, saturation of the smaller fibre by the larger fibre. Evidence for this theory is
Comment [SS19]: Reviewer 1: comment $3 / 4$

Comment [SS18]: Reviewer 1 comment $3 / 4$ 


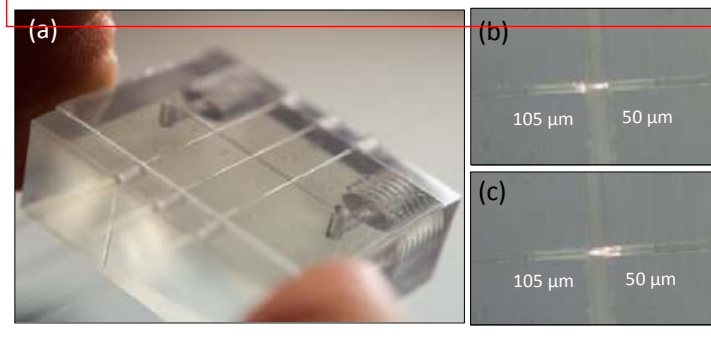

Figure 6. (a) Optimal fibre combination test piece equipped with various combinations of fibres (b) close up images of projection from the $50 \mu \mathrm{m}$ fibre onto the opposing $105 \mu \mathrm{m}$ fibre and (c) projection of $105 \mu \mathrm{m}$ fibre onto opposing $50 \mu \mathrm{m}$

supported by the observation that the transmission efficiency was noticeably higher when the light source was coupled to the $50 \mu \mathrm{m}$ fibre, allowing it to project onto the $105 \mu \mathrm{m}$ fibre than the reverse orientation. In order to demonstrate this, microscopy was used in order to visualise the differences between the transmissions of these configurations - Figure 6 .

As predicted, the larger core sizes demonstrated significant losses as a result of a wider projection of light, whereas the smaller fibre projecting onto the face of a larger fibre appeared to show a much more direct coupling. In the case of the two equal sized fibres, the 50-50 $\mu \mathrm{m}$ combination exhibited the lowest transmission intensity of all the combinations. This was due to the lower amount of light transmitted by the smaller core diameter meaning that losses due to divergence and scattering are more significant than in the case of the larger 105-105 $\mu \mathrm{m}$ combination. It is also possible that equal sized fibre pairs have more stringen coupling requirements and therefore, are more likely to exhibit coupling losses. As a result of these observations, the 50-105 $\mu \mathrm{m}$ was determined to be the optimal configuration for this particular setup.

Through this testing it was therefore possible, at minimal cost (ca. $f 7$ with room for significant reduction via design optimisation), to develop a cell which could be tailored specifically to the equipment and materials available with the potential to test several different combinations of factors in order to realise the most optimal design configuration. Using traditional methods this is often not possible due to the cost associated with producing a design such as the one used here. Instead, expensive modelling software is employed to estimate the data. This increases both the time and cost associated with the acquirement of this data as well as being predictive based on an input of assumptions as opposed to absolute.

\section{Optimal Path Length for Spectroscopic Measurements}

Analysis of nicotinamide solutions demonstrated significantly different linear ranges when path lengths varied from 100 $500 \mu \mathrm{m}$. In this instance the $100 \mu \mathrm{m}$ cell exhibited the widest linear range $(0.5-25.0 \mathrm{mM})$ and the cell featuring a $500 \mu \mathrm{m}$ path length demonstrating the narrowest linear range $(0.5$ $7.5 \mathrm{mM}$ ) - Figure 7. This was attributed to the narrower path

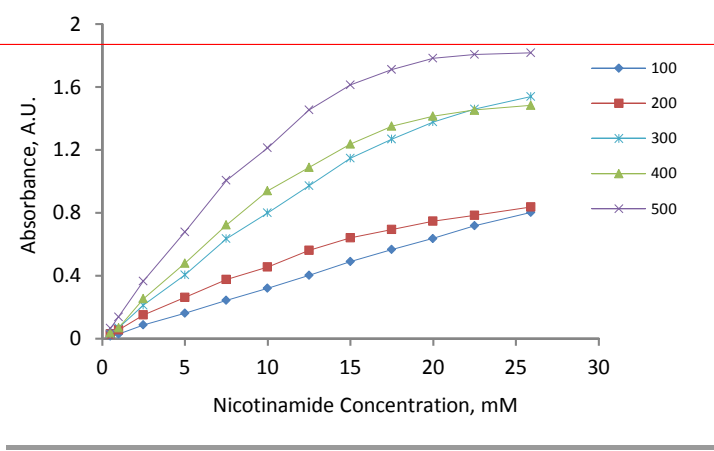

Figure 7. Measured nicotinamide absorbance at varying path lengths

length preventing a loss of collimation for the light exiting the fibre connected to the light source.

Linear regression for the measured absorbance against known concentration values for each path length showed that the $100 \mu \mathrm{m}$ cell was highly linear $\left(R^{2}>0.999\right)$ across the entire range of solutions. This range of concentrations demonstrated by the smaller $100 \mu \mathrm{m}$ cell is ideal for online flow chemistry analysis as a result of the large range of working concentrations possible, acting to increase throughput. This wide range of concentrations is usually not possible using a standard commercial UV-Vis flow cell as a result of the significantly larger path lengths $(1-10 \mathrm{~mm})$. As a result, these systems are often used in detection of low concentration species as opposed to having the ability to monitor both low and high concentrations for quantification and optimisation, as these cells can be.

The maximum working concentration for each cell was estimated by calculating the Pearson product moment correlation coefficient for absorbance values $\leq 1.0$ only. The highest absorbance that led to an $\mathrm{R}^{2}>0.99$ was then taken as the working limit for the cell. As well as establishing the upper working limit for all of these cells, the lower working concentration limits were also established using an aqueous dilution series of Fluorescein in an identical manner. The results of this analysis are located below in Table 4.

As expected, the longer path lengths demonstrated the highest performance in the analysis of lower concentration analytes. However the $100 \mu \mathrm{m}$ cell still produced a useful LOD, especially considering it exhibited such a high upper working concentration limit. These smaller path lengths are therefore capable of measuring solutions from $47 \mu \mathrm{M}$ to $c a .38 \mathrm{mM}$, a significant advantage for online reaction monitoring. However from the data obtained in these studies, an appropriate concentration range can be selected and optimised based upon the concentration ranges gathered. The LOD values obtained using this work are on par or better than previous studies using similar techniques but manufactured using much more complex traditional manufacturing methods. ${ }^{36,37,34}$

The realisation that the $100 \mu \mathrm{m}$ path length produced significantly more linear calibration curves, even at the lower concentration ranges, mean that it is ideally suited to online
Comment [SS21]: Images enlarged and altered in order to improve clarity as mentioned by reviewer 1 in his general comments

Comment [SS22]: Reviewer 1, comment 10 
Table 4. Upper working limits for nicotinamide solutions and the limit of detection for fluorescein solutions in 3DP cells of varying path lengths

\begin{tabular}{|c|cc|}
\hline Path Length, $\mu \mathrm{m}$ & Upper Working limit, $\mathrm{mM}$ & Limit of Detection, $\mu \mathrm{M}$ \\
\hline 100 & $25.9^{*}$ & 47 \\
200 & 15 & 23 \\
300 & 12.5 & 16 \\
400 & 10 & 12 \\
500 & 7.5 & 9 \\
\hline
\end{tabular}

*Upper limit not reached - estimated to be $38 \mathrm{mM}$ by Beer Lambert for absorbance value of 1

flow chemistry analysis of compounds with moderate to low molar attenuation coefficients. This indicated that through the use of high-resolution surface channels, increased reaction information could be obtained over a wide concentration range. This would not have been possible using larger internal channels with inserted fittings due to the requirement for significantly larger path lengths.

This direct monitoring approach contrasts traditional analysis methods in which a 'black-box' approach is taken. In these instances, data is obtained via off-line product analysis without the real-time acquisition of the reaction information from inside the device. This set-up is still not as ideal as in-situ integration where spectroscopic measurements are focused directly inside the microchannel. ${ }^{12-14}$ This direct approach usually comes with the drawback of excessive cost associated with device manufacture or a reduction in portability as a result of large optical set ups. The method used in this work negates both of these issues due to the low cost of the device and the ability to incorporate small, low cost optics directly within the walls of the microchannels, without the need for UV-Vis transparent waveguides.

The ability to produce these multiple iterations of the 3D UV-Vis flow cell has demonstrated the potential of 3DP in the production of microfluidic devices with online reaction monitoring capabilities. Through this advantageous property, the optimal path length for a particular combination of optical fibres was quickly achieved, something not possible when using traditional manufacturing techniques as a result of time and cost restraints. In light of this, a novel flow reaction involving the formation of carvone semicarbazone was performed and optimised online using the $100 \mu \mathrm{m}$ path length UV-Vis cell.

\section{Online Monitoring and Optimisation of Carvone Semicarbazone}

A complex 3D device was manufactured featuring a complex network of internal mixing channels, followed by a high resolution surface channel region for online monitoring Figure 8. This device was able handle multiple experimental runs (upwards of 11 runs made for DOE) without the requirement to change the overlying adhesive layer, fibres or device. It is important to note that whilst no channel degradation was observed in this solvent system, small degrees over extended use are likely. This however should not lead to issues with spectroscopic measurements as the fibres

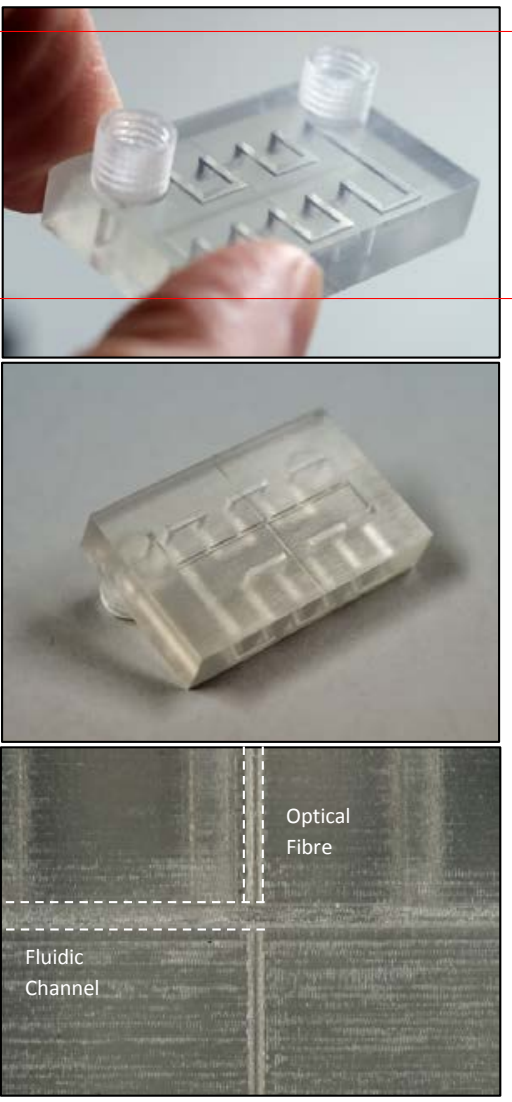

Comment [SS25]: One image remove and all remaining images enlarged. This addresses reviewer 1's general comments on image clarity

Comment [SS23]: Upper and lowe working limits of cells combined into a single table
Figure 8. 3D serpentine mixing device used in optimisation (a) top (b) bottom (c) close up of monitoring region showing fibers intersecting fluidic channel

remain fixed in position by the fibre guides, and therefore the path length can be kept constant through the devices life time. Furthermore the low cost and rapid manufacture of the devices mean that replacement of such components do not carry a significant penalty. The ability to continuously monitor the reaction made identification of the steady state conditions quick and simple. The experiments returning the lowest and highest response at $265 \mathrm{~nm}$ are shown in Figure 9. These correspond to the least forcing and most forcing conditions used in the DOE respectively.

Analysis of the experiments was performed in MODDE software (V 11.0.1.1878, Umetrics, Malmö, Sweden). A model was fitted using multiple linear regression and all terms were left in the model. The resulting $R^{2}, Q^{2}$, model validity and reproducibility were all $>0.90$. Generation of a contour response plot shows the optimum conditions for higher absorbance at $265 \mathrm{~nm}$ are longer residence time and higher temperatures - Figure 9.
Comment [SS24]: Reviewer 3:

comment 1 


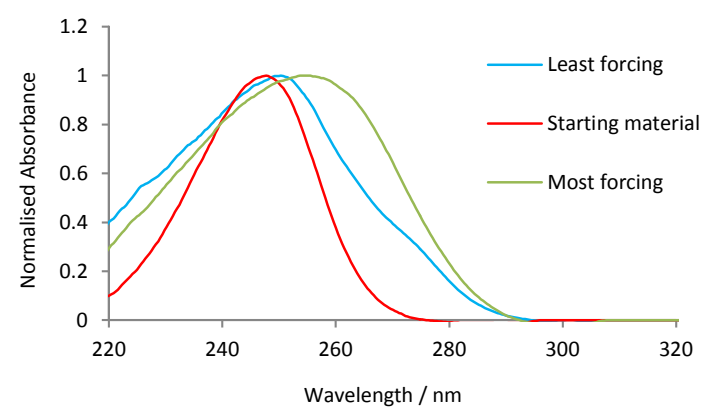

Figure 9. Graph demonstrating the lowest and highest response of the proceeding reaction at the $\lambda_{\max }$ of the emerging product $(265 \mathrm{~nm})$

Analysis of the experiments was performed in MODDE software (V 11.0.1.1878, Umetrics, Malmö, Sweden). A model was fitted using multiple linear regression and all terms were left in the model. The resulting $R^{2}, Q^{2}$, model validity and reproducibility were all $>0.90$. Generation of a contour response plot shows the optimum conditions for higher absorbance at $265 \mathrm{~nm}$ are longer residence time and higher temperatures (Figure 10). As the contour plot demonstrates, the reaction shows an increase in absorption at the $265 \mathrm{~nm}$ wavelength with a corresponding increase in both temperature and residence time. Neither of these reaction condition changes adversely affected the 3DP LOC device, which continued to perform even when exposed to these higher flow rates and/or elevated temperatures. This type of DOE coupled with online monitoring via embedded fibres and UV analysis shows how reactions can be quickly and efficiently assessed, moving towards optimised conditions in a fast and reliable manner.

The reusability and re-configurability of the devices, which stems from the simple integration of optical fibres via PSA tapes, allows for a low cost, semi-disposable and portable device. This is a major advantage to those looking at using LOC technology in a research or prototyping capacity. These devices can be altered by the user in regards to both the type of fibre included (geometry, wavelength, single/multimode etc.) and reactor design to suit the reaction/analyte. This acts to significantly increase the range of potential applications and the information which can be gained from a single device and can allow for custom device manufacture for a specific reaction/analyte at low cost. This method can be extended to multiple monitoring points for multistep reactions in a single device, based upon information gained from earlier test device iterations (e.g. path length, residence time, optimal fibre combination)

\section{Conclusions}

The production of Lab-on-a-Chip (LOC) devices featuring integrated optics for spectroscopic measurements often suffers from expensive and complex manufacturing processes.

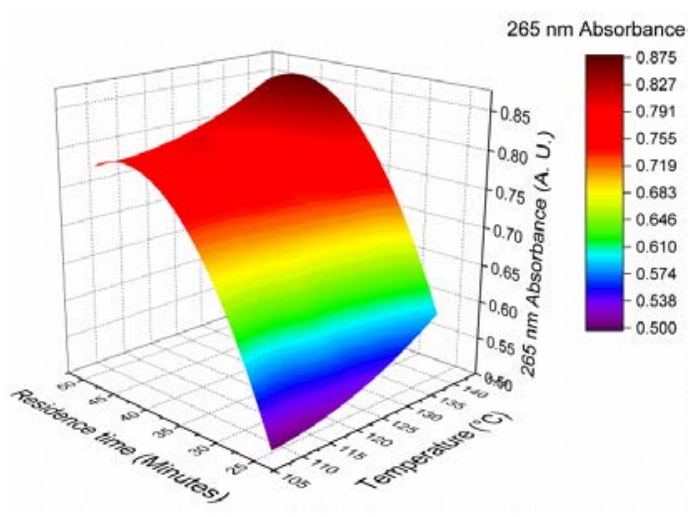

Figure 10. Response contour plot for carvone semicarbazone optimisation

This inevitably reduces the users design freedom and ability to tailor the device to their desired configurations/reaction. 3DPLOC production provides a much simpler and cost effective manufacturing method. By moving away from traditional lithographic techniques, the manufacturing of the devices can be achieved in a single run of the SL system producing several devices with no significant time penalty. This leads to both reductions in cost as well as time. Furthermore the design freedom afforded by this technique allows for fully 3D devices to be manufactured in a fully automated manner, making it suitable for mass customised production.

This work demonstrates 3DP for the production of high resolution LOC devices featuring an integrated optical detection platform. This system centres on guided optics for use with in-line UV-Vis spectroscopy, directly within the fluid channels of the device and was shown to be highly adept at reaction monitoring. The channels successfully formed in the sensing region of these devices represent the highest resolution microfluidic channels formed via SLA to date 150 $\mu \mathrm{m})$. This was made possible through a combination of producing channels on the surfaces of the LOC devices as well as the choice to seal the device using a Lexan cover layer equipped with PSA tapes.

Through the use of pre-aligned, U-shaped channels, two types of optical fibres (differing only in core size) were monolithically integrated directly into the walls of a microfluidic channel without the need for the addition of coupling waveguides, allowing for a more efficient coupling of optical fibres. Furthermore the design freedom of the SL system allowed for the optimum path length separating these fibres for a particular compound to be established through iterative design, acting to increase the effectiveness of the UVVis cell. The absorption detection capabilities of the 3DP LOC devices were demonstrated through absorption measurements of a dilution series of nicotinamide at varying path lengths. Through these designs, the efficiency of the cell was optimised in order to produce a device with high degrees of linearity across a wide range of concentrations as well as
Comment [SS26]: Reviewer 1, comment 5 - regarding the effect of organic solvent on the devices

Comment [SS27]: Reviewer 1comment 2 
low limits of detection. The linearity demonstrated in the case 9 of the $100 \mathrm{\mu m}$ cell very high over a wide range of concentrations, extending up to $c a .30 \mathrm{mM}$, whilst the limit of detection using strongly absorbing compounds was comparable or better than works manufactured using traditional methods. ${ }^{36,37,34}$ The information gained from these initial trails was then used successfully monitor online and optimise the formation of carvone semicarbazone with respect to reaction temperature and residence time in a complex 3DPLOC device. Due to the high number of pharmaceutically important compounds which exhibit chromophores within their structures, these cells could be used to detect or monitor a wide range of species providing the reaction conditions are suitable for the polymer based cells. ${ }^{38}$ Furthermore, the integrated optical fibres would also be ideally suited for cell/particle counting and sizing ${ }^{39-42}$ as well as nanoparticle detection and characterization. ${ }^{43}$

With the increase in funding from various research bodies as well as investment from the private sector, technologies are continuously evolving, fabrication costs are decreasing and the properties of the manufactured parts are becoming ever better. ${ }^{44}$ Material choices for SL are constantly evolving, with biocompatible resins and resins containing a ceramic component available, further adding to the potential applications for this technology in LOC technologies. Merely producing a dormant device via 3DP technology, which provides the user with no feedback has obvious disadvantages and does not represent an evolution of the field or an improvement of what is already available. However, if the full advantages of 3DP technology are utilised, and sensors are integrated into fully 3D devices, it has the potential to make a significant impact on the LOC sector.

\section{Acknowledgements}

This work was supported by the Engineering and Physical Science Research Council (EPSRC) via the Centre for Innovative Manufacturing in Additive Manufacturing.

\section{References}

1 K. F. Jensen, Chem. Eng. Sci., 2001, 56, 293-303.

2 K. Jähnisch, V. Hessel, H. Löwe and M. Baerns, Angew. Chem. Int. Ed. Engl., 2004, 43, 406-46.

3 R. L. Hartman and K. F. Jensen, Lab Chip, 2009, 9, 2495507.

4 S. Marre and K. F. Jensen, Chem. Soc. Rev., 2010, 39, 1183202.

5 S. Löbbecke, in Micro Process Engineering. Fundamentals, Devices, Fabrication, and Applications, ed. N. Kockmann, Wiley-VCH Verlag GmbH, 2006, 249-266.

6 V. Sans and L. Cronin, Chem. Soc. Rev., 2016, 45, 20322043.

7 J.-S. Park, K.-B. Park, K.-S. Shin, H.-D. Park, M.-C. Kim, J.-R Kim, S.-J. Park and Y.-H. Song, Sensors Actuators B Chem., 35 2006, 117, 516-522.

8 F. B. Myers and L. P. Lee, Lab Chip, 2008, 8, 2015-2031.
K. Swinney and D. J. Bornhop, Electrophoresis, 2000, 21 1239-1250.

D. Janasek, J. Franzke and A. Manz, Nature, 2006, 442, 374-380.

T. Kitamori, M. Tokeshi, A. Hibara and K. Sato, Anal. Chem. 2004, 76, 52A-60A

12 J. T. Adeosun and A. Lawal, Chem. Eng. Sci., 2010, 65, 1865-1874.

13 R. Barzin, S. R. A. Shukor and A. L. Ahmad, Sensors Actuators B Chem., 2010, 146, 403-409.

14 J.-M. Commenge, T. Obein, G. Genin, X. Framboisier, S. Rode, V. Schanen, P. Pitiot and M. Matlosz, Chem. Eng. Sci. 2006, 61, 597-604.

15 H. Lu, M. A. Schmidt and K. F. Jensen, Lab Chip, 2001, 1 22-28.

S. Krawczyk, Phys. status solidi, 2003, 998-1012.

A. Waldbaur, H. Rapp, K. Länge and B. E. Rapp, Anal. Methods, 2011, 3, 2681-2716.

18 G. Comina, A. Suska and D. Filippini, Lab Chip, 2014, 14, 2978-2982.

19 J. Do, J. Y. Zhang and C. M. Klapperich, Robot. Comput. Integr. Manuf., 2011, 27, 245-248.

20 I. K. Dimov, L. Basabe-Desmonts, J. L. Garcia-Cordero, B. M. Ross, Y. Park, A. J. Ricco and L. P. Lee, Lab Chip, 2011, 11, 845-850.

21 J. L. Erkal, A. Selimovic, B. C. Gross, S. Y. Lockwood, E. L. Walton, S. McNamara, R. S. Martin and D. M. Spence, Lab Chip, 2014, 14, 2023-2032.

22 V. Dragone, M. H. Rosnes, V. Sans, P. J. Kitson and L. Cronin, Lab Chip, 2012, 12, 3267-3271.

23 A. K. Au, W. Lee and A. Folch, Lab Chip, 2014, 14, 1294301.

24 P. Tseng, C. Murray, D. Kim and D. Di Carlo, Lab Chip, 2014, 14, 1491-1495.

25 B. C. Gross, J. L. Erkal, S. Y. Lockwood, C. Chen and D. M. Spence, Anal. Chem., 2014, 86, 3240-3253.

26 A. J. Capel, S. Edmondson, S. D. R. Christie, R. D. Goodridge, R. J. Bibb and M. Thurstans, Lab Chip, 2013, 13, 45834590.

27 S. Waheed, J.-M. Cabot Canyelles, N. Macdonald, R. M Guijt, T. Lewis, B. Paull and M. C. Breadmore, Lab Chip, 2016, 16, 1993-2013.

28 Data sheet for Accura 60 SL material http://www.3dsystems.com/sites/www.3dsystems.com/fil es/24075-s12-04-a-sds-ghs-english-accura-60.pdf.

29 R. J. Jackman, T. M. Floyd, R. Ghodssi, M. A. Schmidt and K. F. Jensen, J. Micromechanics Microengineering, 2001, 11 263-269.

30 F. Benito-Lopez, W. Verboom, M. Kakuta, J. H. G. E. Gardeniers, R. J. M. Egberink, E. R. Oosterbroek, A. van den Berg and D. N. Reinhoudt, Chem. Commun., 2005, 28572859.

31 T. Monaghan, A. J. Capel, S. D. Christie, R. A. Harris and R. J. Friel, Compos. Part A Appl. Sci. Manuf., 2015, 76, 181-193. D. F. Swinehart, J. Chem. Educ., 1962, 39, 333-335.

J. Miller and J. Miller, Statistics and chemometrics for analytical chemistry, 2005, $6^{\text {th }}$ Edition, 124-128.

L. Ceriotti, K. Weible, N. F. de Rooij and E. Verpoorte, Microelectron. Eng., 2003, 67-68, 865-871.

A. I. Shallan, P. Smejkal, M. Corban, R. M. Guijt and M. C. Breadmore, Anal. Chem., 2014, 86, 3124-3130.
Comment [SS28]: Reviewer 3 Comment 3 
L. Malic and A. G. Kirk, Sensors Actuators A Phys., 2007, 135, 515-524.

37 A. Prabhakar and S. Mukherji, Lab Chip, 2010, 10, 748-754.

38 R. Porta, M. Benaglia and A. Puglisi, Org. Process Res. Dev., 2015, 20, 2-25.

39 A. L. Givan, Methods Mol. Biol., 2011, 699, 1-29.

40 K. E. H. and A. R. S. Sun, N. Sergeev, J. Francis, Y. Kostov, M. Yang, H. A. Bruck, ed. K. E. H. and A. Rasooly, Horizon Scientific Press, 2009, 1, 161.

41 N. Pamme, R. Koyama and A. Manz, Lab Chip, 2003, 3, 187-192.

42 B. H. Weigl, R. L. Bardell and C. R. Cabrera, Adv. Drug Deliv. Rev., 2003, 55, 349-377.

43 J. Yue, F. H. Falke, J. C. Schouten and T. A. Nijhuis, Lab Chip, 2013, 13, 4855-4863.

44 A. Hernandez and K. V. Wong, ISRN Mech. Eng., 2012, 110. 